\title{
Mapeo Vivencial del Fenómeno Enseñanza - Aprendizaje en el Espacio de Aula Escolar - El Caso de Uruguay
}

Sensorial Mapping of the Teaching - Learning Phenomena in the Classroom Space - The Case of Uruguay

\author{
> Paula Cardellino \\ Universidad ORT Uruguay, Uruguay \\ Universidad del Bío-Bío, Chile \\ cardellino@ort.edu.uy
}

\author{
$>\quad$ Claudio Araneda \\ Universidad del Bío-Bío, Chile \\ madpro@ubiobio.cl
}

\begin{abstract}
Children are entitled to equal access to education; this applies to the classroom setting. The classroom size, shape and layout are commonly taken for granted and little consideration is taken to how this affects the learning experience. This paper argues that the traditional classroom environment does not allow for equal interaction between teachers and pupils. Preliminary findings of a case study in 4 classrooms in Uruguay are presented. The conclusions suggest that not all pupils in the classroom receive quality interaction with the teacher. A more comprehensive approach to designing spaces that are conducive to learning will benefit this interaction.
\end{abstract}

Keywords: Mapeo vivencial; Aula escolar; Interacción; Proxemia; Diseño arquitectónico.

\section{Introducción}

Existe una clara inquietud global por mejorar la accesibilidad y calidad de la educación y, en particular, el de optimizar la infraestructura educacional. Al mismo tiempo, los establecimientos actuales presentan una uniformidad formal basada en condiciones constructivas, con escasas referencias a particularidades funcionales, culturales y/o locales. Recientemente, se dispone de nuevos medios de registro y estrategias de análisis vivencial, que han demostrado ser aportes en otras áreas, que pueden ser útiles para diagnosticar el desempeño actual de espacios e identificar condiciones de diseño que contribuyan a un mejor desempeño futuro. Por tanto, la aplicación de estos medios ofrece una nueva perspectiva de la relación espacio/usuario. Por ejemplo, diferencias sustanciales de percepción según la localización del alumno dentro del aula que contradicen las sugerencias de equidad planteadas por las declaraciones de los derechos del niño (UNICEF, 2004).

La investigación tiene como objetivo primordial sentar bases metodológicas para el desarrollo de un diagnóstico vivencial del espacio aula en relación al fenómeno enseñanza/aprendizaje. Este artículo presenta resultados preliminares de cuatro casos de estudio en aulas en escuelas en Uruguay con respecto a la esfera visual, considerada clave en el proceso de enseñanza/aprendizaje y que constituye parte de la tesis doctoral.

\section{Fenómeno enseñanza/aprendizaje y proxemia}

Desde una concepción de la enseńanza como hecho comunicativo determinado por la relación maestro-estudiante y estudiante-estudiante, Hall (1969) plantea que las condiciones espaciales tales como: la arquitectura, la iluminación y ventilación, los ruidos, el clima, la distribución del mobiliario en el aula y, también, las relaciones de espacio entre las mesas y los asientos de los alumnos entre sí, y con el maestro, tienen una incidencia, tanto positiva como negativa, en la comunicación y la enseńanza. De este modo, se reconoce que aspectos no verbales son significativos dentro del ámbito escolar en el que, constante y necesariamente, se interactúa.

La enseñanza escolar es un proceso basado en la comunicación directa, la que implica comunicación verbal y no verbal, de forma simultanea entre alumnos y maestros donde el canal verbal es considerado necesario e irremplazable (Woods, 2013). Un número significativo de investigaciones señalan la importancia del diseño espacial en la calidad de la comunicación y la enseñanza (Higgins et al., 2005; Horne-Martin, 2004; Woolner, 2010). Sin embargo, las aulas actuales exhiben una uniformidad en el diseño, principalmente en su forma y proporciones, que no ha sido alterada, o al menos cuestionada, desde la Revolución Industrial hace 200 años a pesar de los cambios dramáticos en la sociedad y la economía (Leiringer y Cardellino, 2011). Esto dicho, la escasez de investigaciones en el área no se corresponde con la importancia de este fenómeno (Lim et al. 2012; MacPherson, 1984; Marx et al., 2000). Si se ha de coincidir con el hecho de que la educación es efectivamente un derecho del nińo y que por tanto deben tener acceso a ella en igualdad de condiciones, la investigación en esta área debe intensificarse. 
En la segunda mitad del siglo XX, el antropólogo Americano Edward T. Hall desarrolla el concepto de proxemia. Desde sus estudios seminales concernientes con el impacto que el espacio tiene sobre el comportamiento humano se sabe que el espacio hecho por el hombre (arquitectónico y urbano) influye directamente en su comportamiento así como en las relaciones humanas que dentro de él se desarrollan (Hall, 1973). Sin embargo hasta la fecha, su instrumentalización ha sido exigua y dispersa. Aunque numerosos son los estudios proxemicos realizados en el campo de la educación, menos frecuentes son las investigaciones que utilizan este concepto para analizar los espacios de aula desde un punto de vista alternativo. De esta forma, se busca actualizar el impulso proxemico incursionando en el estudio del aula como espacio de comunicación/interacción entre individuos donde las distancias personales juegan un papel importante para un adecuado proceso de aprendizaje.

En su trabajo seminal, Hall (1966) introduce rangos de distancia y define 4 categorías: pública, social-consultativa, casual-personal e íntima, de acuerdo con las distancias establecidas de forma espontanea entre el emisor y el receptor de un determinado mensaje. En el contexto del aula, la mayoría de la comunicación se realiza en el espacio social-consultativo, ya que constituye la relación formal apropiada entre maestro y alumno (figura 1).

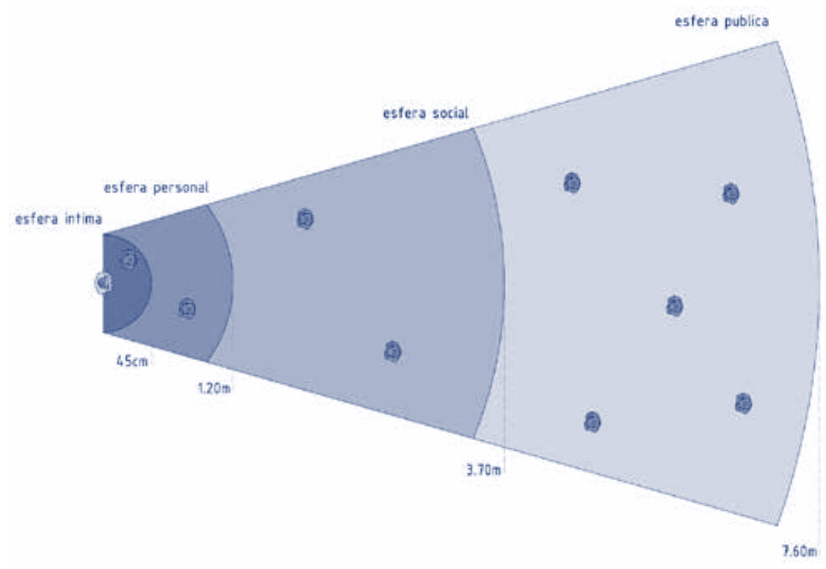

Figura 1: Distancias proxemicas derivadas de Hall (1969).

El espacio aula que se estudia en esta investigación es típico de un salón de clase tradicional, donde la mayoría del tiempo la actividad de la maestra se sitúa al frente de la clase, junto al pizarrón, $\mathrm{y}$ donde los alumnos se sientan en filas mirando hacia este lugar.

\section{Casos, metodologia y análisis}

Las cuatro aulas estudiadas se ubican en escuelas de Uruguay. Dos de las aulas están situadas en una escuela pública que responde a la modalidad de Escuela de Tiempo Completo (ETC). El edificio donde se encuentran se inauguró en el año 2013 siguiendo la normativa de diseño establecida por la iniciativa de las ETC y se encuentra localizada en una de las zonas del país donde el nivel socio económico es considerado bajo. Estas aulas (aula 1 y aula 2) albergan 26 alumnos y un maestro respectivamente. Las dimensiones y proporciones del espacio de estas aulas son similares, tendiendo a la forma cuadrada (figura 2 y figura 3 ).

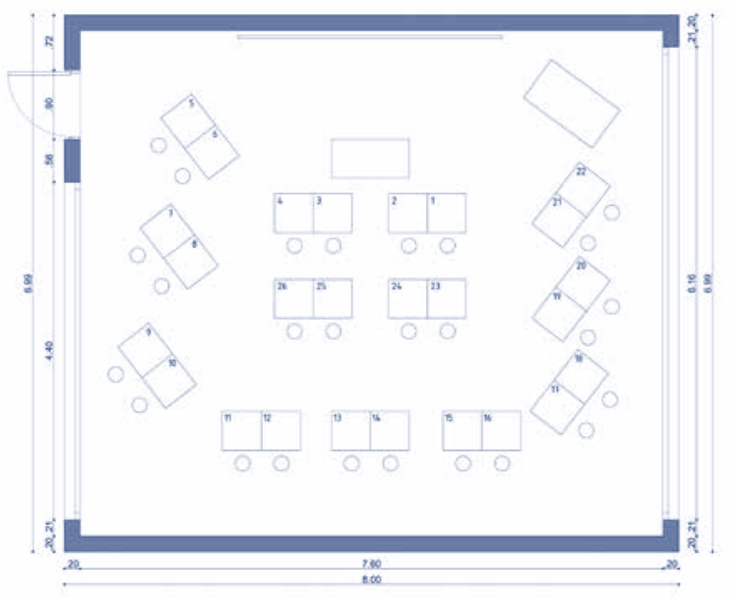

Figura 2: Planta aula 1 con ubicación de alumnos.



Figura Э: Planta aula 2 con ubicación de alumnos.

Las otras dos aulas están localizadas dentro de un colegio privado en Montevideo considerado de clase media-alta. El edificio que las alberga fue inaugurado en 1955 siguiendo las normativas establecidas en esa época. Estas aulas albergan 30 alumnos cada una con 1 maestro a cargo. La forma de estas aulas es rectangular, con 8 metros de profundidad y 6 metros de ancho (figura 4 y figura 5).

La estrategia metodológica utilizada para realizar el mapeo vivencial de cada alumno en relación con su interacción/comunicación con la maestra deriva de (1) estudios de isovistas, (2) distancias derivadas de la proxemia e (3) información fotográfica obtenida in situ (isovista vivencial). De la planta isovista, se extrae información relativa a las posibilidades de comunicación directa con la maestra que tiene cada alumno desde su lugar en el espacio aula. De la planta de distancias proxemicas, derivadas de la teoría de Hall, se verifica la esfera en la que cada alumno se encuentra con respecto a la maestra dentro de la clase. Por último, el análisis de la isovista o campo visual (fotográficamente abstraído) que tiene, de forma efectiva, cada alumno de su maestra. Para obtener esta última, cada 
alumno realizó una captura desde su asiento en dirección al pizarrón y la maestra. Con esta información se construye una ficha personal para cada alumno. Esta ficha informa sobre el estado de la isovista y campo visual efectivo de cada alumno y además, sobre el estado de la relación maestra alumno desde el punto de vista proxémico.



Figura 4: Planta aula 3 con ubicación de alumnos.

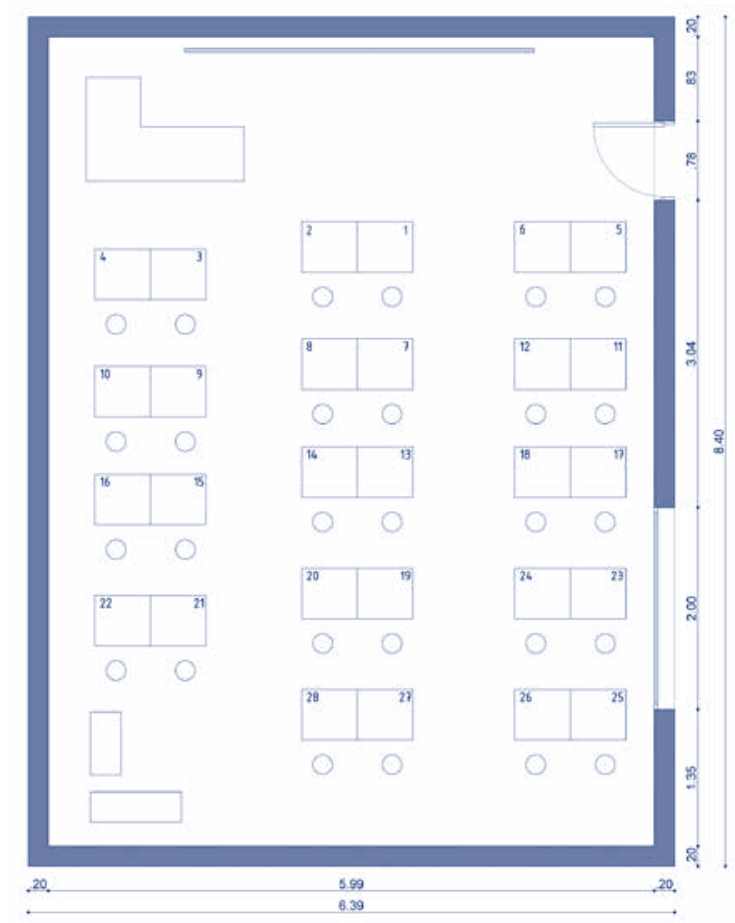

Figura 5: Planta aula 4 con ubicación de alumnos.

\section{Resultados preliminares}

Se constata que los alumnos no están en igualdad de condiciones con respecto a la fuente de información (maestra y/o pizarrón) desde el punto de vista de la isovista y el campo visual. Un alto porcentaje de campos visuales de alumnos esta compuesta de cabezas y cuerpos de otros alumnos que se interponen entre la maestra y el alumno en distintos grados. Esto sugiere que la calidad de la relación alumno - maestra se deteriora tan pronto como aparecen segundas filas. A su vez, esta situación se agrava en la medida en que se superponen mas filas.

Por su parte, la superposición de información proxémica sobre la planta del aula sugiere que la organización de filas y la profundidad de estas afecta tanto la comunicación verbal como la no verbal entre los alumnos y la maestra pues al mismo tiempo, la distancia afecta la percepción visual mutua. Se considera que los alumnos que se encuentran situados en la zona pública son los que se encuentran comprometidos con respecto a la comunicación con la maestra.

\section{Implicancias para el diseño}

El corpus metodológico diseńado y aplicado en este trabajo proporciona evidencia de que aspectos cotidianos, y como tales no cuestionados tales como las filas, juegan un rol protagónico en la experiencia diaria que el niño tiene en el aula. Específicamente, en la calidad de la relación visual que establece con la maestra y el pizarrón, las dos fuentes de conocimiento tradicionales del aula escolar. En otras palabras, comienza a explicar y entregar evidencia de porque no es lo mismo sentarse en la primera fila que en la segunda, tercera o cuarta. La importancia de estos descubrimientos podrían arrojar luz y objetividad sobre fenómenos tan importantes y claves como la cantidad máxima ideal de alumnos por clase.

\section{Conclusiones}

La investigación sugiere que un importante número de alumnos en el aula uruguaya no reciben el tipo de interacción necesaria con el maestro, en especial en el área de la comunicación visual. Se sugiere, por lo tanto, un enfoque más integral y versátil del diseño de los espacios de aprendizaje que propicien una educación acorde con los principios universales que gobiernan la formación de todo niño y que, finalmente, beneficie los resultados de aprendizaje y, con ello, la calidad de la formación que reciben los alumnos.

\section{Agradecimientos}

El trabajo presentado aquí es parte de la investigación que se lleva a cabo en la Facultad de Arquitectura, Universidad ORT Uruguay en el marco del Doctorado en Arquitectura y Urbanismo que la autora realiza en la Universidad del Bío-Bío, Chile.

\section{Referencias}

Hall, E. (1969). The hidden dimension. New York: Anchor Books. Hall, E. (1973). The Silent Language. New York: Anchor Books. 

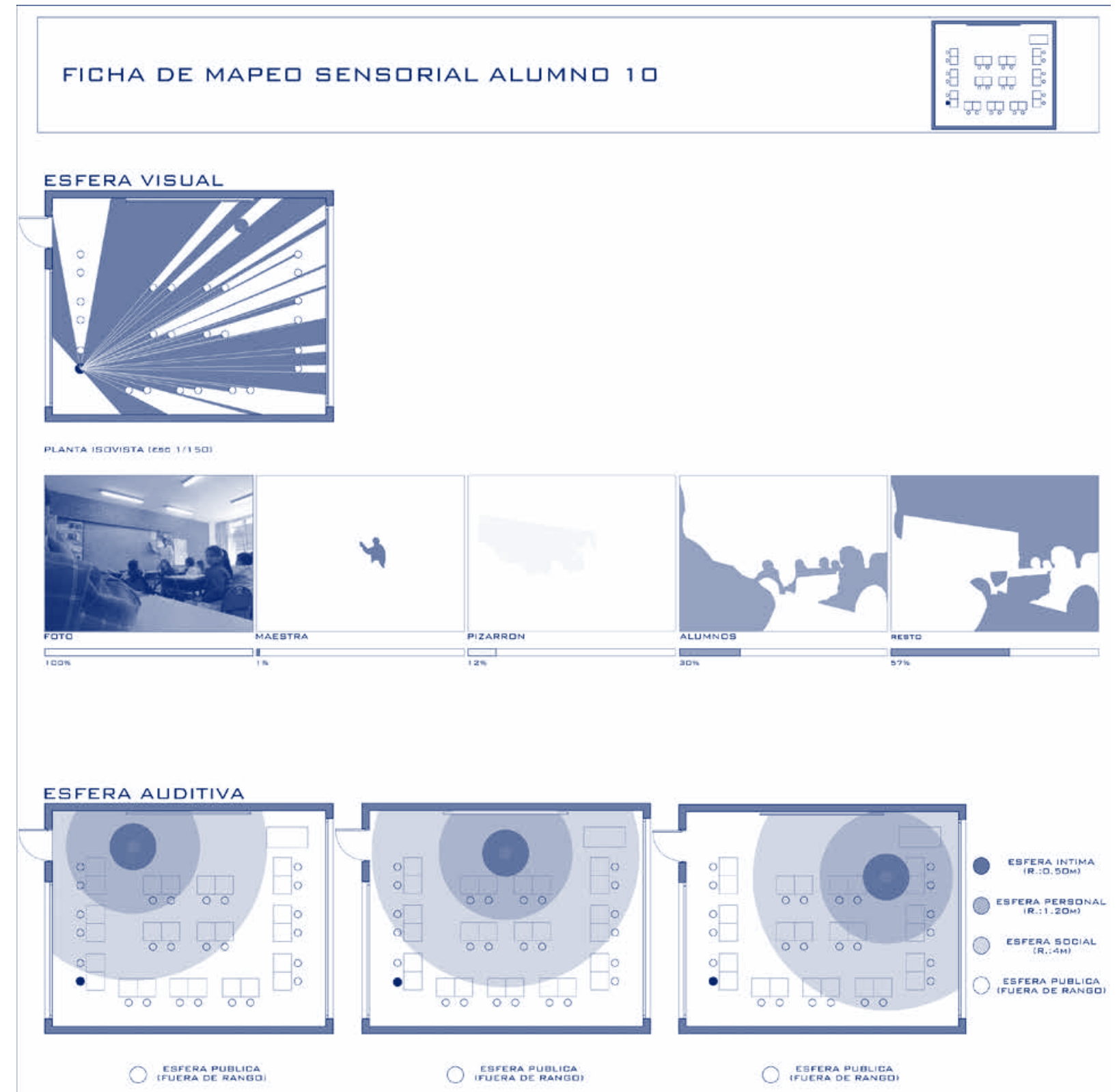

Figura 6: Ejemplo de ficha de mapeo sensorial de un alumno.

Higgins, S., Hall, E., Wall, K., Woolner, P. y McCaughey, C. (2005) The impact of school environments: a literature review. London: Design Council.

Horne-Martin, S. (2004). The classroom environment and children's performance - is there a relationship?, In Spencer, C. y Blades, M. (eds) Children and their Environments. Learning, using and designing spaces, Cambridge: Cambridge University.

Leiringer, R. y Cardellino, P. (2011): Schools for the twenty-first century: school design and educational transformation. British Educational Research Journal, 37(6), 915-934.

Lim, F. V., O’Halloran, K. L., \& Podlasov, a. (2012). Spatial pedagogy: mapping meanings in the use of classroom space.
Cambridge Journal of Education, 42(2), 235-251.

MacPherson, J. C. (1984). Environments and Interaction in Row-and-Column Classrooms. Environment and Behavior, 16(4), 481-502.

Marx, A., U. Fuhrer y T. Hartig (2000). Effects of Classroom Seating Arrangements on Children's Question-asking, Learning Environments Research, 2, 249-263.

UNICEF (2004) The universal decalaration of human rights. Turkey.

Woolner, P. (2010). The design of learning spaces. London: Continuum.

Wood, J (2013) Interpersonal Communication: Everyday Encounters. Boston: Wadsworth. 